\title{
ChanNEl MAXimization IN Wireless BACKHAUL BASED HETNETS
}

\author{
Mostafa Hefnawi \\ Department of Electrical and Computer Engineering, Royal Military College of Canada, \\ Kingston, Canada
}

\begin{abstract}
In this paper, we consider the optimization of wireless capacity-limited backhaul links in future heterogeneous networks (HetNets). We assume that the HetNet is formed with one macro-cell base station $(M B S)$, which is associated with multiple small-cell base stations (SBSs). It is also assumed both the MBS and the SBSs are equipped with massive arrays, while all mobiles users (macro-cell and small-cell users) have single antenna. For the backhaul links, we propose to use a capacity-aware beamforming scheme at the SBSs and MRC at the MBS. Using particle swarm optimization (PSO), each SBS seeks the optimal transmit weight vectors that maximize the backhaul uplink capacity and the access uplinks signal-tointerference plus noise ratio (SINR). The performance evaluation in terms of the symbol error rate (SER) and the ergodic system capacity shows that the proposed capacity-aware backhaul link scheme achieves similar or better performance than traditional wireless backhaul links and requires considerably less computational complexity.
\end{abstract}

\section{KEYWORDS}

HetNets, wireless backhaul, cognitive radio, Massive MIMO, multiuser MIMO, PSO.

\section{INTRODUCTION}

Recently, deploying small cell networks over existing macro-cellular networks, also known as heterogeneous networks (HetNets), has emerged as a promising solution to deal with the increasing wireless traffic demands in next generation $5 \mathrm{G}$ cellular networks [1]-[6]. The users in these HetNets are offloaded from the congested macro-cell base stations (MBSs) to the small-cell base stations (SBSs), which enhanced their quality of service (QoS) and increase the overall system capacity. These HetNets are supported by Gigahertz bandwidth backhaul links that connect MBSs and the associated SBSs. Such Gigahertz bandwidth can be achieved by conventional optical fiber or millimeter-waves (mmWaves) based wireless backhauls. Optical fiber backhauls wile reliable, they might be expensive and difficult to deploy in HetNets where several small cells are unplanned and installed quite arbitrarily. Wireless backhauls, on the other hand, are more attractive to overcome the restriction of deployment and installation and can provide a cheap and scalable solution. However, to achieve high spectral efficiency in HetNets with wireless backhauling, frequency reuse across the coexisting network tiers (backhaul and access links) is essential and interference management is critical. Cognitive radio based HetNets (CR-HetNets) has emerged as a promising solution that provides a more energy efficient and dynamic way to use the spectrum by enabling small-cell to share licensed bands in opportunistic manner [5]-[6]. In CR-HetNets, macro-cell users, which are considered as primary users (PUs) take the priority to access the channels, whereas small-cell users, which are considered as secondary users (SUs), can access the channels as long as the corresponding PUs do not use them. However, most of these proposed CR-HetNets have assumed opportunistic spectrum sharing which may not be reliable and may limit the system capacity since it suffers from the 
interruptions imposed by the primary network (PN) on the SUs who must leave the licensed channel when PUs emerge. Also, with opportunistic spectrum sharing, SUs can still cause interference to PUs due to their imperfect spectrum sensing. In cellular systems, one way to overcome these limitations is to incorporate multiuser multi-input multi-output (MU-MIMO) approach into cognitive radio networks (CRNs) to achieve higher spectral efficiency by multiplexing multiple SUs on the same time-frequency resources and protecting PNs from SUs' interferences. MU-MIMO techniques have been successfully deployed in $4 \mathrm{G}$ cellular systems for traditional fixed spectrum assignment (FSA) approaches [7]-[15] and a vast number of multiuser detection algorithms are presently being tailored towards solving the MU-MIMO processing in cognitive networks by imposing additional constraints to protect licensed users' QoS[22]-[16]. More specifically, capacity-aware MU-MIMO schemes have been proposed for both FSA [13][15] and CR networks [16]-[17] using different multiuser detections schemes such as maximum ratio combining (MRC) and minimum mean-squared error (MMSE), and have shown the potential to exhibit better system capacity and provide better SER enhancement than traditional singular value decomposition (SVD)-based MU-MIMO systems. On the other hand, it was shown that the use of large-scale antenna arrays (also called massive MIMO) could achieve tremendous boost of MU-MIMO systems system performance [23]-[26]. In this paper, therefore, we will be applying the concept of MU massive MIMO and CR in HetNets. We assume that the MBSs and SBSs are equipped with massive arrays, while all mobiles users have single antenna. We deploy two MU-MIMO schemes, namely, MRC at the access link (SUs to SBSs) and capacityaware/MRC at the backhaul link (SBSs to MBS). Such a system can significantly improve the system performance in terms of link reliability, spectral efficiency, and energy efficiency. It can also achieve optimal performances with the simplest forms of user detection techniques, i.e., MRC [12]. On the other hand, most of the proposed capacity-aware MU-MIMO schemes require the use of gradient search algorithms in order to solve the constrained optimization problem in CRNs [16]-[17]. These techniques become very computationally expensive in large-scale MIMO systems because of the vast amounts of baseband data that are generated and require the constrained optimization problem to be differentiable. Thus, in our capacity-aware backhaul link scheme we will be exploring free-derivative population-based training algorithms such as the particle swarm optimization (PSO) that are well known by their simple/fast hardware implementation. PSO was initially introduced by Kennedy and Eberhart in [27] and has received a lot of attention in recent years. It is an evolutionary computation technique inspired by swarm intelligence such as fish schooling and bird flocking looking for the best food spot (exploring the optimal solution) in the search space where a quality measure, fitness, can be evaluated without any a priori knowledge. The PSO algorithm in this paper will be used at the backhaul link to seek iteratively the transmit beamforming weights of each SBS that maximize the uplink (UL) MIMO backhaul channel capacity. Under the assumption of very large number of antennas at the SBSs and the MBS, we derive semi-analytic expressions for the symbol error rate (SER) and the ergodic channel capacity, which quantify the reliability and spectral efficiency of the MU-MIMO based HetNet. The derived expressions are then validated with Monte-Carlo simulation and used to evaluate the performance of the proposed PSO-based capacity-aware (PSO-CA) backhaul link. The contribution of this paper includes the extension of the cognitive capacity-aware massive MU-MIMO schemes to wireless backhaul links and the development of semi-analytical model for the SER and channel capacity analyses in HetNets.

\section{SYSTEM MODEL}

We consider the UL access scenario shown in Fig. 1 of a HetNet with $K$ small cells and one macro cell that share the same frequency band. Each small cell includes one SBS equipped with massive $N$-element antenna array and $L_{s}$ single-antenna secondary users (SUs). Each SBS and its users act as a cognitive networkthatcoexist, via concurrent spectrum access, with $L_{p}$ macro-cell primary users (PUs) and their primary MBS, which is also equipped with massive $M$-element 
antenna array. It is also assumed that both the SBS and the MBS detect independent OFDM data streams from their mobile users simultaneously on the same time-frequency resources.

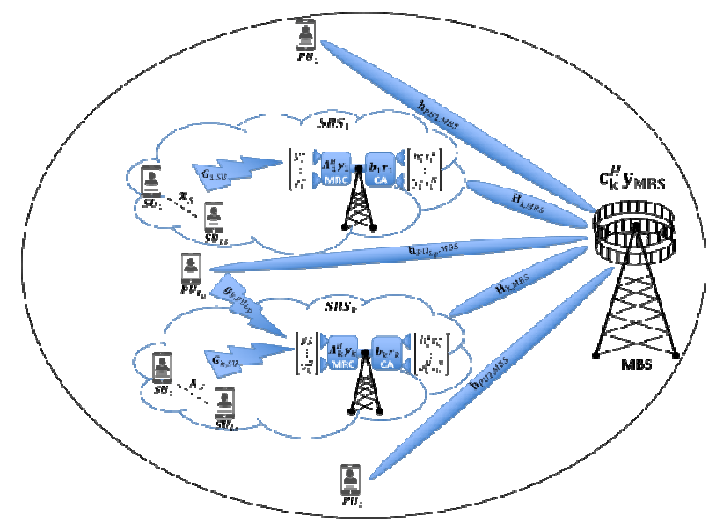

Figure 1. System Model: HetNet consisting of one macro-cell and $K$ small-cells and their corresponding users.

Let $\boldsymbol{x}_{s}\left[f_{i}\right]=\left\{x_{1}^{S}, x_{2}^{S}, \cdots, x_{L_{S}}^{S}\right\}$ and $\boldsymbol{x}_{p}\left[f_{i}\right]=\left\{x_{1}^{p}, x_{2}^{p}, \cdots, x_{L_{p}}^{p}\right\}$ denote, respectively, the set of $L_{s}$ SUs signals and $L_{p}$ PUs signals transmitted on each subcarrier, $f_{i}, i=1, \cdots, N_{c}$, where $N_{c}$ denotes the number of subcarriers per OFDM symbol in the system.The analysis is done separately on each subcarrier. For brevity therefore, we drop the frequency index $f_{i}$.

\subsection{Access link}

The $\mathrm{N} \times 1$ received signal vector at the $k^{\text {th }} \mathrm{SBS}$ is given by

$\boldsymbol{y}_{k}=\sqrt{p_{u}} \boldsymbol{G}_{k, S U} \boldsymbol{x}_{S}+\boldsymbol{n}_{k, S B S}+\mathbf{I}_{P U, S B S}$

where $\boldsymbol{G}_{k, S U} \in \mathbb{C}^{\mathrm{N} \times L_{S}}$ is the channel matrix between the $k^{\text {th }}$ SBS and its $L_{S}$ users, $\boldsymbol{x}_{s} \in \mathbb{C}^{L_{s} \times 1}$ is the transmittedsignal vector of $L_{s}$ users in the $k^{\text {th }}$ small-cell, $p_{u}$ is the average power transmitted by each user (Here we assume equal power allocation for all users), $\boldsymbol{n}_{k, S B S} \in \mathbb{C}^{\mathrm{N} \times 1}$ is the received AWGNvector at the SBS, and $\mathbf{I}_{P U, S B S}$ represents the interference introduced by macrocell users (PUs) at the SBS, and is given by

$\mathbf{I}_{P U, S B S}=\sqrt{p_{p}} \boldsymbol{G}_{k, P U} \boldsymbol{x}_{p}$

Where $\boldsymbol{G}_{k, P U} \in \mathbb{C}^{\mathrm{N} \times L_{p}}$ is the channel matrix between the $\mathrm{k}^{\text {th }}$ SBS and $L_{p}$ users, $p_{p}$ is the average power transmitted by each PU, and $\boldsymbol{x}_{p} \in \mathbb{C}^{L_{p} \times 1}$ is the transmittedsignal vector of $L_{p}$ users in the HetNet.

For the uplink access link, we consider MRC detection scheme at each SBS. The $k^{\text {th }}$ SBS processes its received signal $\boldsymbol{y}_{k}$ by multiplying it by the $\mathrm{N} \times L_{s}$ receive beamforming weight matrix $\boldsymbol{A}_{k}^{H}$ as follows

$$
\boldsymbol{r}_{k}=\boldsymbol{A}_{k}^{H} \boldsymbol{y}_{k}=\sqrt{p_{u}} \boldsymbol{A}_{k}^{H} \boldsymbol{G}_{k, S U} \boldsymbol{x}_{s}+\boldsymbol{A}_{k}^{H} \boldsymbol{n}_{k, S B S}+\boldsymbol{A}_{k}^{H} \mathbf{I}_{P U, S B S}
$$

The detection of user $l_{s}$ by its $k^{\text {th }}$ SBS can then be expressed as 
$r_{k, l_{s}}=\boldsymbol{a}_{k, l_{s}}^{H} \boldsymbol{y}_{k}=\sqrt{p_{u}} \boldsymbol{a}_{k, l_{s}}^{H} \boldsymbol{G}_{k, S U} \boldsymbol{x}_{s}+\boldsymbol{a}_{k, l_{s}}^{H} \boldsymbol{n}_{k, S B S}+\boldsymbol{a}_{k, l_{s}}^{H} \mathbf{I}_{P U, S B S}$

Where $r_{k, l_{s}}$ is the $l_{s}^{\text {th }}$ element of $\boldsymbol{r}_{k}$ and $\boldsymbol{a}_{k, l_{s}}$ is the $l_{s}^{\text {th }}$ column of $\boldsymbol{A}_{k}$.

\subsection{Backhaul link}

For the backhaul link, the expression for the array output of the MBS in Fig. 1 can be written for each subcarrier as

$\mathbf{y}_{\mathrm{MBS}}=\sum_{k=1}^{K} \mathbf{H}_{k, M B S} \boldsymbol{b}_{k} \boldsymbol{r}_{k}+\mathbf{n}_{M B S}+\mathbf{I}_{P U, M B S}$,

where $\mathbf{y}_{\mathbf{M B S}}$ is the $M \times 1$ vector containing the outputs of the $M$-element array at the MBS, $\mathbf{H}_{k, M B S}$ is the $M \times N$ frequency-domain channel matrix representing the transfer functions from the $N$-element antenna array of the $\mathrm{k}^{\text {th }}$ SBS to the $M$-element antenna array of the MBS, $\boldsymbol{b}_{k}=\left[b_{1}, b_{2} \quad \ldots, b_{N}\right]^{T}$ is the $N \times 1$ complex transmit weight vector of the $k^{\text {th }}$ SBS, $\mathbf{n}_{M B S}$ is the received $M \times 1$ complex additive white Gaussian noise vector at the MBS, and $\mathbf{I}_{P U, M B S}[\mathrm{k}]$ represents the interference introduced by PUs to SUs at the MBS and is given by

$\mathbf{I}_{P U, M B S}=\sqrt{p_{p}} \mathbf{H}_{P U, M B S} \boldsymbol{x}_{p}$

Where $\mathbf{H}_{P U, M B S}$ is the $M \times L_{\mathrm{p}}$ channel matrix from the $L_{p}$ PUs to the MBS's $M-$ element antenna array.

The MBS detects the $k^{\text {th }}$ SBS data by multiplying the output of the array $\mathbf{y}_{\text {MBS }}$ with the $M \times$ 1receiving weight vector, $\mathbf{c}_{\mathrm{k}}^{H}$ as follows

$\hat{x}_{k}=\mathbf{c}_{\mathrm{k}}^{H} \mathbf{y}_{\mathrm{MBS}}=\mathbf{S}_{\mathrm{k}}+\mathbf{S}_{I_{S}}+\mathrm{S}_{I_{p}}+\mathbf{N}$

Where

$\mathbf{S}_{\mathrm{k}}=\mathbf{c}_{\mathrm{k}}^{H} \mathbf{H}_{k} \boldsymbol{b}_{k} \boldsymbol{r}_{k} \quad$ is the signal detected from the $\mathrm{k}^{\text {th }} \mathrm{SBS}$,

$\mathbf{S}_{\boldsymbol{I}_{\boldsymbol{s}}}=\mathbf{c}_{\mathbf{k}}^{H} \sum_{i=1, i \neq k}^{K} \mathbf{H}_{i} \boldsymbol{b}_{i} \boldsymbol{r}_{i}$ is the multiple-access interference (MAI) from the $K-1$ other SBSs, $\mathbf{S}_{I_{p}}=\sqrt{p_{p}} \mathbf{c}_{\mathrm{k}}^{H} \mathbf{H}_{P U, M B S} \boldsymbol{x}_{p}$ is the MAI from $\mathrm{L}_{p}$ PUs, and $\mathbf{N}=\mathbf{c}_{\mathrm{k}}^{H} \mathbf{n}_{M B S}$ is the noise signal at the array output of the MBS,

For the backhaul link, it is assumed that each SBS is transmitting with a capacity-aware beamforming scheme that will be discussed in Section 4 and that the MBS is detecting SBSs' signals using MRC scheme.

\section{Symbol ERror Rate ANd ERgodic Channel CAPACITY}

The symbol error rate, $S E R_{k, l_{s}}$, associated with $l_{s}{ }^{\text {th }}$ user of the $k^{\text {th }}$ SBS can be expressed as $S E R_{k, l_{s}}=E_{\gamma_{k, l_{s}}}\left[a Q\left(\sqrt{2 \mathrm{~b} \gamma_{k, l_{s}}}\right)\right]$,

WhereE [.] denotes the expectation operator, $Q\left(\right.$.) denotes the Gaussian Q-function, $\gamma_{k, l_{s}}$ is the signal-to-interference-plus-noise ratio (SINR) associated with the $l_{s}^{\text {th }}$ user of the $k^{\text {th }}$ SBS , and $a$ and $b$, are modulation-specific constants. For binary phase shift keying (BPSK), $a=1$ and $b=$ 1 , for binary frequency shift keying (BFSK) with orthogonal signaling $a=1$ and $b=0.5$, while for M-ary phase shift keying (M-PSK) $a=2$ and $b=\sin ^{2}(\pi / M)$. 
Using (7), the signal detected from the $l_{s}^{\text {th }}$ user of the $k^{\text {th }}$ SBS can be expressed by (9) and the signal detected by the MBS from the $l_{s}{ }^{\text {th }}$ user of the $k^{\text {th }}$ SBS can be expressed by (10).

$$
\begin{gathered}
\mathbf{S}_{\mathrm{k}, l_{s}}=\mathbf{c}_{\mathrm{k}}^{H} \mathbf{H}_{k, M B S} \boldsymbol{b}_{k} r_{k, l_{s}}=\mathbf{c}_{\mathrm{k}}^{H} \mathbf{H}_{k, M B S} \boldsymbol{b}_{k} \boldsymbol{a}_{k, l_{s}}^{H} \boldsymbol{y}_{k} \\
=\mathbf{c}_{\mathrm{k}}^{H} \mathbf{H}_{k, M B S} \boldsymbol{b}_{k} \boldsymbol{a}_{k, l_{s}}^{H} \boldsymbol{G}_{k, S U} \boldsymbol{x}_{S}+\mathbf{c}_{\mathrm{k}}^{H} \mathbf{H}_{k, M B S} \boldsymbol{b}_{k} \boldsymbol{a}_{k, l_{s}}^{H} \boldsymbol{n}_{k, S B S}+\mathbf{c}_{\mathrm{k}}^{\mathrm{H}} \mathbf{H}_{k, M B S} \boldsymbol{b}_{k} \boldsymbol{a}_{k, l_{s}}^{H} \mathbf{I}_{P U, S B S} \\
\widehat{\boldsymbol{x}}_{k, l_{S}}=\mathbf{c}_{\mathrm{k}}^{H} \mathbf{y}_{\mathrm{MBS}}=\mathbf{S}_{\mathrm{k}, l_{s}}+\mathbf{S}_{I_{S}}+\mathbf{S}_{I_{p}}+\mathbf{N}
\end{gathered}
$$

The SINR at the MBS for user $l_{s}$ of the $k^{\text {th }}$ SBS can thus be depicted as

$$
\gamma_{k, l_{s}}=\frac{\mathbf{c}_{\mathrm{k}}^{H} \mathbf{H}_{k, M B S} \square_{k} \boldsymbol{a}_{k, l_{s}}^{H} \boldsymbol{G}_{k, S U} \boldsymbol{G}_{k, S U}^{H} \boldsymbol{a}_{k, l_{s}} \boldsymbol{b}_{k}^{H} \mathbf{H}_{k, M B S}^{H} \boldsymbol{c}_{k}}{\mathbf{c}_{\mathrm{k}}^{H} \mathbf{B}_{k} \boldsymbol{c}_{k}}
$$

Where $\mathbf{B}_{k}$ is the covariance matrix of the interference-plus-noise, and is given by

$$
\mathbf{B}_{k}=\mathbf{B}_{S B S}+\mathbf{B}_{P U, M B S}+\mathbf{B}_{P U, S B S}+\mathbf{B}_{n}
$$

Where

$$
\begin{aligned}
& \mathbf{B}_{\mathrm{SBS}}=\sum_{i=1, i \neq k}^{K} \mathbf{H}_{i, M B S} \boldsymbol{b}_{i} \boldsymbol{r}_{i} \boldsymbol{r}_{i}^{H} \boldsymbol{b}_{i}^{H} \mathbf{H}_{i, M B S}^{H} \\
& \mathbf{B}_{\mathrm{PU}, \mathrm{MBS}}=p_{p} \mathbf{H}_{M B S, l_{p}} \mathbf{H}_{M B S, l_{p}}^{H} \\
& \mathbf{B}_{\mathrm{PU}, \mathrm{SBS}}=p_{p} \mathbf{H}_{k, M B S} \boldsymbol{b}_{k} \boldsymbol{a}_{k, l_{s}}^{H} \boldsymbol{G}_{k, P U} \boldsymbol{G}_{k, P U}^{H} \boldsymbol{a}_{k, l_{s}} \boldsymbol{b}_{k}^{H} \mathbf{H}_{k, M B S}^{H} \\
& \mathbf{B}_{\mathrm{n}}=\mathbf{c}_{\mathrm{k}}^{H} \mathbf{c}_{\mathrm{k}}+\mathbf{H}_{k, M B S} \boldsymbol{b}_{k} \boldsymbol{a}_{k, l_{s}}^{H} \boldsymbol{a}_{k, l_{s}} \boldsymbol{b}_{k}^{H} \mathbf{H}_{k, M B S}^{H}
\end{aligned}
$$

We observe that in general, the off diagonal elements of $\mathbf{B}_{k}$ are non-zero, reflecting the color of the interference. However, in the asymptotic case of large $M$-element array, and given equal power transmitted by all users, the central limit theorem (CLT) can be invoked to show that [13],

$$
\mathbf{B}_{k}=\left(\frac{(K-1) p_{u}+L_{p} p_{p}}{\sigma_{n}^{2}}+1\right) \sigma_{n}^{2} \mathbf{I}_{M} .
$$

Also, for a large-scale MIMO, the channel vectors are nearly-orthogonal and hence $\boldsymbol{G}_{k, S U} \boldsymbol{G}_{k, S U}^{H}$ and $\mathbf{H}_{k, M B S} \mathbf{H}_{k, M B S}^{H}$ can be approximated by

$$
\begin{aligned}
& \boldsymbol{G}_{k, S U} \boldsymbol{G}_{k, S U}^{H}=\frac{\xi}{r} \boldsymbol{I}_{r} \\
& \mathbf{H}_{k, M B S} \mathbf{H}_{k, M B S}^{H}=\frac{\eta}{v} \boldsymbol{I}_{v}
\end{aligned}
$$

Where $r \triangleq \min \left(\mathrm{L}_{\mathrm{s}}, \mathrm{N}\right), \quad \xi=\sum_{\mathrm{i}=1}^{r} \lambda_{i, l_{s}}$, with $\lambda_{i, l_{s}}$ representing the eigenvalues of $\boldsymbol{G}_{k, S U} \boldsymbol{G}_{k, S U}^{H}$, $v \triangleq \min (\mathrm{M}, \mathrm{N})$ and $\eta=\sum_{\mathrm{i}=1}^{v} \lambda_{i, \mathrm{k}}$, with $\lambda_{i, \mathrm{k}}$ representing the eigenvalues of $\mathbf{H}_{k, M B S} \mathbf{H}_{k, M B S}^{H}$.

Thus the SINR $\gamma_{k, l_{s}}$ can be expressed as

$\gamma_{k, l_{s}}=\frac{\xi}{r} \frac{\eta}{v}\left(\frac{\boldsymbol{a}_{k, l_{s}}^{H} \boldsymbol{a}_{k, l_{s}} \boldsymbol{b}_{k} \boldsymbol{b}_{k}^{H}}{(K-1) p_{u}+L_{p} p_{p}+\sigma_{n}^{2}}\right)$

The ergodic channel capacity, per subcarrier, for each SBS $k$ is given by [13]

$\mathrm{C}\left(\mathbf{H}_{k, M B S}, \boldsymbol{b}_{k}\right)=E\left(\log _{2}\left\{\mathbf{I}+\frac{p_{\mathrm{u}}}{N} \frac{\mathbf{H}_{k, M B S} \boldsymbol{b}_{k} \boldsymbol{a}_{k, l_{s}}^{H} \boldsymbol{G}_{k, S U} \boldsymbol{G}_{k, S U}^{H} \boldsymbol{a}_{k, l_{s}} \boldsymbol{b}_{k}^{H} \mathbf{H}_{k, M B S}^{H}}{\mathbf{B}_{k}}\right\}\right)$

WhereE [.] denotes the expectation operator.

By noticing that for asymptotically large $N, \frac{1}{N} \boldsymbol{G}_{k, S U} \boldsymbol{G}_{k, S U}^{H} \rightarrow \boldsymbol{I}_{N}$ almost surely, and using (13) and (15) we can express the channel capacity asymptotically as 


$$
\mathrm{C}\left(\mathbf{H}_{k, M B S}, \boldsymbol{b}_{k}\right)=E\left(\log _{2}\left\{\mathbf{1}+p_{\mathrm{u}} \frac{\eta}{v}\left(\frac{\boldsymbol{a}_{k, l_{s}}^{H} \boldsymbol{a}_{k, l_{s}} \boldsymbol{b}_{k} \boldsymbol{b}_{k}^{H}}{(K-1) p_{u}+L_{p} p_{p}+\sigma_{n}^{2}}\right)\right\}\right)
$$

\section{Capacity-Awarebackhaul Link}

In the proposed capacity-aware backhaul link, the weight vector for the $k^{\text {th }}$ SBS is updated at each iteration $n$ until it reaches the optimal beamforming vector, $\left(\boldsymbol{b}_{i}\right)_{\text {opt }}$, that maximizes the ergodic backhaul channel capacity for each SBSkofthe HetNet. This channel capacity can be expressed, at each iteration $n$, by:

$\mathrm{C}(\mathrm{n})=E\left(\log _{2}\left\{\mathbf{1}+p_{\mathrm{u}} \frac{\eta}{v}\left(\frac{\boldsymbol{a}_{k, l_{s}}^{H} \boldsymbol{a}_{k, l_{s}} \boldsymbol{b}_{k}(n) \boldsymbol{b}_{k}^{H}(n)}{(K-1) p_{u}+L_{p} p_{p}+\sigma_{n}^{2}}\right)\right\}\right)$

To maximize the backhaul link capacity we propose to employ particle swarm optimization algorithm where particles are mapped to the transmit beamforming and fly in the search space, aiming to maximize the fitness function given by the channel capacity of (19). First, the PSO generates $Z$ random particles for each SBS (i.e., random weight vector $\boldsymbol{b}_{\mathrm{k}}^{(z)}, z=1, \ldots, Z$ of length $N \times 1$ ) to form an initial population set $\mathrm{S}$ (swarm). The algorithm computes the channel capacity according to (14) for all particles $\boldsymbol{b}_{\mathrm{k}}^{(z)}$ and then finds the particle that provides the global optimal channel capacity for this iteration, denoted $\boldsymbol{b}_{\mathrm{k}}^{(z, g b e s t)}$. In addition, each particle $z$ memorizes the position of its previous best performance, denoted $\boldsymbol{b}_{\mathrm{k}}^{(z, p b e s t)}$. After finding these two best values, PSO updates its velocity $\boldsymbol{v}_{\mathrm{k}}^{(z)}$ and its particle positions $\boldsymbol{w}_{\mathrm{k}}^{(z)}$ at each iteration $n$ using (20) and (21), respectively, where $c_{1}$ and $c_{2}$ are acceleration coefficients towards the personal best position (pbest) and/or global best position (gbest), respectively, $\varphi_{1}$ and $\varphi_{2}$ are two random positive numbers in the range of $[0,1]$, and $\omega$ is the inertia weight which is employed to control the exploration abilities of the swarm.

$\boldsymbol{v}_{\mathrm{k}}^{(z)}(n+1)=\boldsymbol{\omega} \boldsymbol{v}_{\mathrm{k}}^{(z)}(n)+\boldsymbol{c}_{\mathbf{1}} \boldsymbol{\varphi}_{\mathbf{1}}\left(\boldsymbol{b}_{\mathrm{k}}^{(z, p b e s t)}(n)-\boldsymbol{b}_{\mathrm{k}}^{(z)}(n)\right)+\boldsymbol{c}_{\mathbf{2}} \boldsymbol{\varphi}_{\mathbf{2}}\left(\boldsymbol{b}_{\mathrm{k}}^{(z, g b e s t)}(n)-\boldsymbol{b}_{\mathrm{k}}^{(z)}(n)\right)$

$\boldsymbol{b}_{\mathrm{k}}^{(z)}(n+1)=\boldsymbol{b}_{\mathrm{k}}^{(z)}(n)+\boldsymbol{v}_{\mathrm{k}}^{(z)}(n+1)$

Large inertia weights will allow the algorithm to explore the design space globally. Similarly, small inertia values will force the algorithms to concentrate in the nearby regions of the design space. This procedure is repeated until convergence (i.e., channel capacity remains constant for a several number of iterations or reaching maximum number of iterations). An optimum number of iterations is tuned and refined iteratively by evaluating the average number of iterations required for PSO convergence as a function of the target MSE for algorithm termination and as a function of the population size. Since random initialization does not guarantee a fast convergence, in our optimization procedure we consider that the initial value of $\boldsymbol{b}_{\mathrm{k}}^{(z)}(n)$ at iteration index $n=0$ is given by the eigen-beamforming (EBF) weight, i.e., $\boldsymbol{b}_{\mathrm{k}}^{(z)}(0)=\sqrt{P_{\boldsymbol{u}}} \mathbf{u}_{\boldsymbol{m a x}, \boldsymbol{k}}$, where $\mathbf{u}_{\max , \boldsymbol{l}_{\boldsymbol{s}}}$ denotes the eigenvector corresponding to $\boldsymbol{\lambda}_{\max , k}$, the maximum eigenvalue of $\mathbf{H}_{k, \mathrm{MBS}}^{H} \mathbf{H}_{k, \mathrm{MBS}}$. This initial guess enables the algorithm to reach a more refined solution iteratively by ensuring fast convergence and allows to compute the initial value of the received beamforming vector at iteration index $n=0$. In our case we assume MRC at the receiving MBS, i.e.:

$\mathbf{c}_{\mathrm{k}}^{H}(0)=\left(\mathbf{B}_{k}\right)^{-\mathbf{1}} \mathbf{H}_{k, M B S} \boldsymbol{b}_{k}(0)$ 


\section{Simulation Results}

In our simulation setups, we consider a HetNet organized into $K$ SBSs $(\mathrm{K}=20)$ and one macrocell. The number of antennas at the SBSs and at the MBS is the same, $N=M$, and is varying from 25 to 200. Each SBS is serving $L_{s}=10$ users and the macro-cell is serving $L_{p}=10$ users, each transmitting with a single antenna. We assume QPSK modulation. For the OFDM configurations, we assume the $256-\mathrm{OFDM}$ system $\left(N_{c}=256\right)$, which is widely deployed in broadband wireless access services. For the backhaul link we assume an MU-MIMO system with capacity-aware beamforming at each SBS and MRC detection at the MBS. For the access link, we assume MRC detection at each SBS. For the PSO parameters, the swarm size is 30, the maximum iteration number is 25 and the acceleration coefficients are $c_{1}=c_{2}=2$.The inertia weight $\omega$ ranges from 0.9 to 0.4 and varies as the iteration goes on.

Fig. 2shows the the system capacity of the proposed PSO-CA using Monte-Carlo and semianalytic methods for different number of antenna at the SBS and MBS. We observe that there is a gap between the Monte-Carlo and the semi-analytical results for $\mathrm{M}=\mathrm{N}=50$, especially at high SNR. This difference is due to (19), which was derived in the asymptotic case of largenumber of antenna. For $\mathrm{M}=\mathrm{N}=200$, however, we noticed that the gap has almost disappeared, which indicated we have approached the asymptotic case.

Fig. 3 shows the system capacity of the proposed PSO-CA and the traditional Eigen-beamforming schemes forM $=N=25$, and 200. It is observed that for both cases PSO-CA is outperforming Eigen-beamforming. It is also noted that as we increase the number of antennas the performance gap between the two schemes is reduced. This means that when the number of base station antennas becomes large, PSO-CA can achieve the same level or better performance than Eigenbeamforming with less computational complexity. Fig. 4, on the other hand, compares the SER performance of both schemes for the same scenario as in Fig. 3. It is observed PSO-CA is outperforming Eigen-beamforming in both cases.

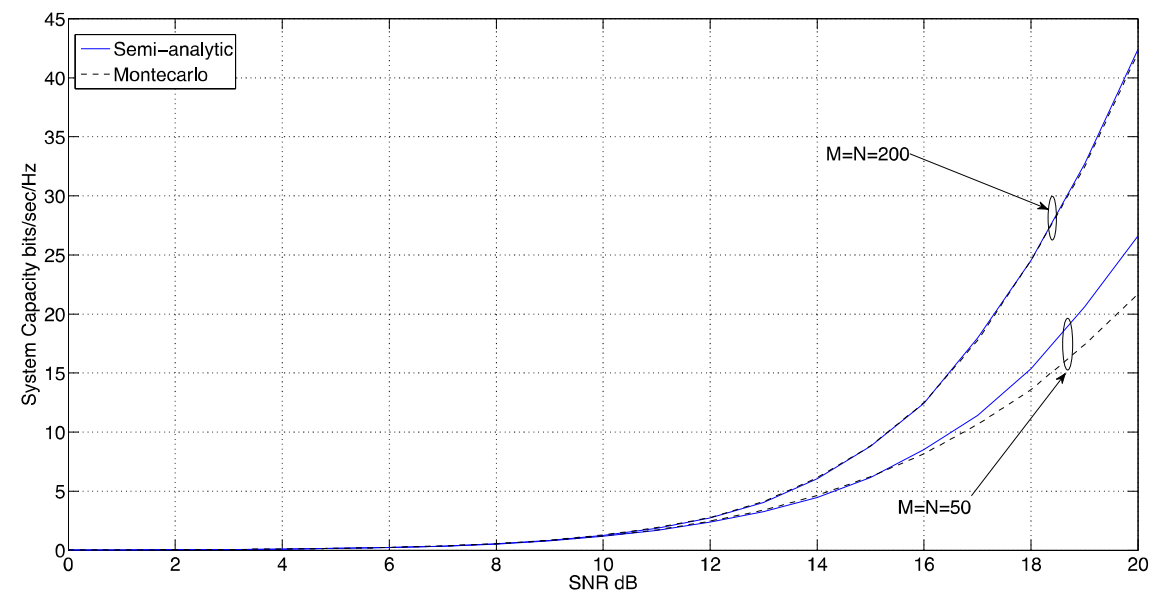

Figure 2.Ergodic channel capacityof HetNet using PSO-CA for $\mathrm{K}=20$ SBSs and $\mathrm{M}=\mathrm{N}=50$ and 200 antennas: Monte-Carlo vs semi-analytic. 


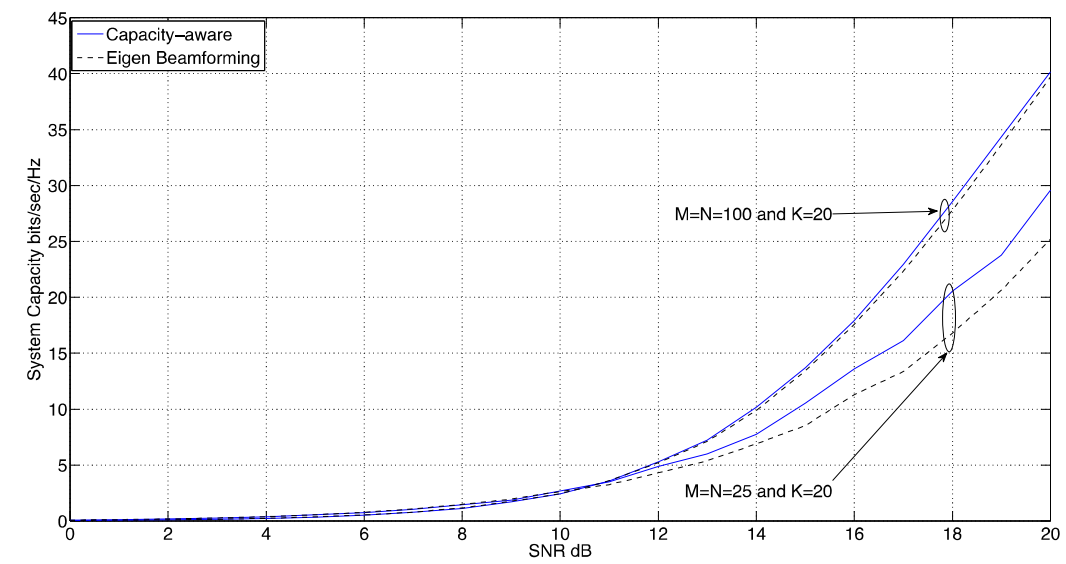

Figure 3.Ergodic channel capacityof HetNet using PSO-CA and Eigen-beamforming schemes for $\mathrm{K}=20$ SBSs and $\mathrm{M}=\mathrm{N}=25$ and 100 antennas.

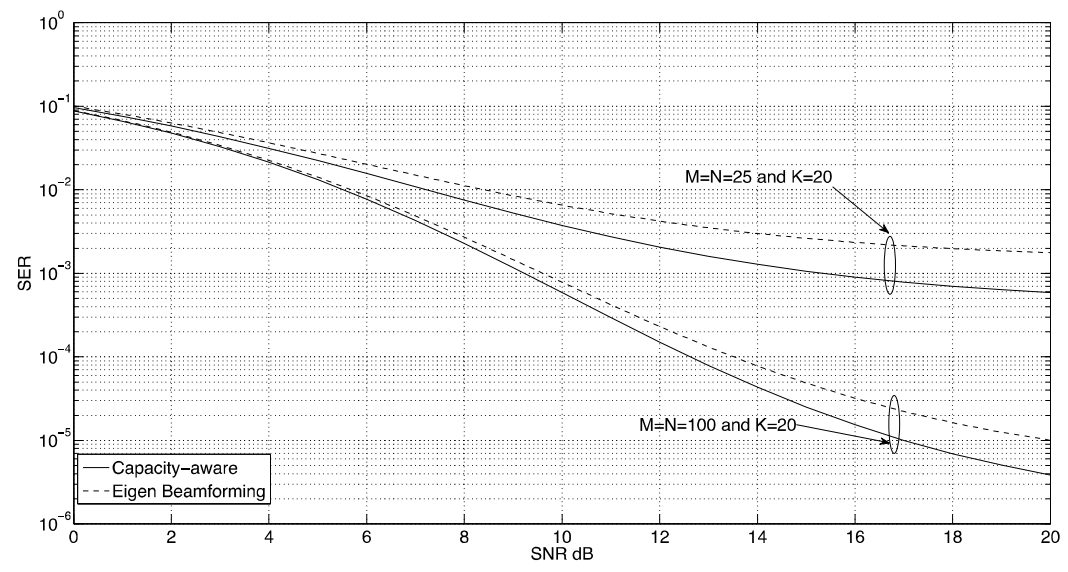

Figure 4.SER performance of HetNet using PSO-CA and Eigen-beamforming schemes for K=20 SBSs and $\mathrm{M}=\mathrm{N}=25$ and 100 antennas.

\section{CONCLUSION}

This paper proposes a capacity-aware wireless backhaul link where cognitive small cells communicate with a MBS using a PSO-based large-scale multiple-input multiple-output (LSMIMO) beamforming scheme. The proposed algorithm iteratively seeks the optimal transmit weight vectors that maximize the channel capacity of each SBS in the HetNet. It was shown that the proposed system is able to achieve a low computational complexity (without requiring an inverse of the covariance matrix) with the same level or better performance than the convectional eigen-beamforming.

\section{ACKNOWLEDGEMENTS}

The author would like to thank the Canadian MicroelectronicsCorporation (CMC) for providing the Heterogeneous Parallel Platform to run the computationally-intensive Monte-Carlo Simulations 
International Journal of Wireless \& Mobile Networks (IJWMN) Vol. 9, No. 3, June 2017

\section{REFERENCES}

[1] U. Siddique, H. Tabassum, E. Hossain, and D. I. Kim, "Wireless backhauling of 5G small cells: Challenges and solution approaches," IEEE Wireless Communications, Special Issue on "Smart Backhauling and Fronthauling for 5G Networks", vol. 22, no. 5, Oct. 2015, pp. 22-31.

[2] Zhen Gao, Linglong Dai, De Mi, Zhaocheng Wang, Muhammad Ali Imran, and Muhammad Zeeshan Shakir, "MmWave Massive MIMO Based Wireless Backhaul for 5G Ultra-Dense Network," IEEE Wireless Communications, vol. 22, no. 5, pp. 13-21, Oct. 2015.

[3] H. Tabassum, A. Hamdi Sakr, and E. Hossain, "Analysis of massive MIMO-enabled downlink wireless backhauling for full-duplex small cells," IEEE Transactions on Communications, vol. 64, no. 6, June 2016, pp. 2354-2369.

[4] Mehrdad Shariat, Emmanouil Pateromichelakis, Atta ul Quddus, and Rahim Tafazolli, "Joint TDD Backhaul and Access Optimization in Dense Small-Cell Networks," IEEE Transactions on Vehicular Technology, vol. 64, no. 11, November 2015, pp. 5288-5299.

[5] H. ElSawy, E. Hossain, and D. I. Kim, "HetNets with cognitive small cells: User offloading and Distributed channel allocation techniques," IEEE Communications Magazine, Special Issue on "Heterogeneous and Small Cell Networks (HetSNets)", vol. 51, no. 6, June 2013.

[6] Zhi Yan, Wentao Zhou, Shuang Chen, and Hongli Liu, "Modeling and Analysis of Two-Tier HetNets with Cognitive Small Cells," IEEE Access, 2016.

[7] M. Y. Alias, A. K. Samingan, S. Chen, and L. Hanzo, "Multiple antenna aided OFDM employing minimum bit error rate multiuser detection," IEE Electron. Lett., Vol. 39, No 24, pp. 1769-1770, Nov. 2003.

[8] P. Vandenameele, L. Van Der Perre, M. G. E. Engels, B. Gyselinckx, and H. J. De Man, “A combined OFDM/SDMA approach,” IEEE J. Select. Areas Commun., Vol. 18, No 11, pp. 2312-2321, Nov. 2000.

[9] M. Jiang, S. Ng, and L. Hanzo, "Hybrid Iterative Multiuser Detection for Channel Coded Space Division Multiple Access OFDM Systems," IEEE Trans. Veh. Technol., Vol.55, No1, Jan. 2006.

[10] M. Munster and L. Hanzo, "Performance of SDMA Multi-User Detection Techniques for WalshHadamard-Spread OFDM Schemes,” IEEE-VTC'01, Vol. 4, pp. 2319-2323, Oct. 2001.

[11] K.-K. Wong, R. Cheng, K. B. Letaief, and R. D. Murch, "Adaptive Antennas at the Mobile and Base Stations in an OFDM/TDMA System,” IEEE Trans. Commun., Vol. 49, No 1, Jan. 2001.

[12] M. Kang, "A comparative study on the performance of MIMO MRC systems with and without cochannel interference," IEEE Transactions on Communications, Vol. 52 , Iss. 8, pp. 1417 - 1425, 2004.

[13] A. I. Sulyman and M. Hefnawi, "Adaptive MIMO Beamforming Algorithm Based on Gradient Search of the Channel Capacity in OFDMSDMA System," IEEE Commun. Letters, Vol. 12, No. 9, pp. 642644, Sept. 2008.

[14] A. I. Sulyman, and M. Hefnawi, "Performance Evaluation of Capacity-Aware MIMO Beamforming Schemes in OFDM-SDMA Systems," IEEE Trans. Commun., Vol. 58, No. 1, Jan. 2010.

[15] A. I. Sulyman, and M. Hefnawi, "Capacity-Aware Linear MMSE Detector for OFDM-SDMA Systems,” IET Communications, Vol. 4, Iss. 9, June 2010.

[16] M. Hefnawi and A. Abubaker, "Channel Capacity Maximization in Multiuser Large Scale MIMOBased Cognitive Networks", International Journal of Microwave and Optical Technology, Nov. 2014.

[17] M. Hefnawi, “ SDMA Aided Cognitive Radio Networks," IEEE 26th Biennial Symposium on Communications, pp. 10 - 14, 2012.

[18] L.-L. Yang and L.-C. Wang, "Zero-Forcing and Minimum Mean-Square Error Multiuser Detection in Generalized Multicarrier DS-CDMA Systems for Cognitive Radio,” EURASIP Journal on Wireless Communications and Networking, pp. 1-13, 2008.

[19] K. Hamdi, W. Zhang, and K. B. Letaief, “ Opportunistic spectrum sharing in cognitive MIMO wireless networks," IEEE Transactions on Wireless Communications, Vol. 8, No 8, pp. 4098-4109, August 2009.

[20] R. Zhang and Y.-C. Liang. Exploiting multi-antennas for opportunistic spectrum sharing in cognitive radio networks. IEEE Journal of Selected Topics in Signal Processing, Vol. 2, No. 1, pp. 88-102, February 2008.

[21] S. Yiu, M. Vu, and V. Tarokh, "Interference Reduction by Beamforming in Cognitive Networks," IEEE GLOBECOM Telecom. Conf., pp. 1-6, 2008. 
[22] L. Bixio, G. Oliveri, M. Ottonello, M. Raffetto, and C. Regazzoni, “ Cognitive radios with multiple antennas exploiting spatial opportunities," IEEE Transaction on Signal Processing, Vol. 58, No 8, pp. 4453-4459, August 2010.

[23] T. L. Marzetta, "Noncooperative cellular wireless with unlimited numbers of base station antennas," IEEE Tran. Wirel. Comm., vol. 9, no. 11, pp. 3590 - 3600, Nov. 2010.

[24] F. Rusek, D. Persson, B. Lau, E. Larsson, T. Marzetta, O. Edfors, and F. Tufvesson, "Scaling up MIMO: Opportunities and challenges with very large arrays," IEEE Signal Process. Mag., vol. 30, no. 1, pp. 40-60, 2013.

[25] H. Q. Ngo, E. G. Larsson, and T. L. Marzetta, "Energy and spectral efficiency of very large multiuser MIMO systems," IEEE Trans. Commun., vol. 61, no. 4, pp. 1436-1449, Apr. 2013.

[26] J. Hoydis, S. ten Brink, and M. Debbah, "Massive MIMO in the UL/DL of cellular networks: How many antennas do we need?" IEEE J. Sel. Areas Commun., vol. 31, no. 2, pp. 160-171, Feb. 2013.

[27] J. Kennedy, RC. Eberhart, "Particle swarm optimization," Proceedings of the IEEE Conference on Neural Networks IV, , pp. 1942-1948, 1995.

[28] E. Mijangos, "Approximate Subgradient Methods for Lagrangian Relaxation on Networks," in System Modeling and Optimization,. IFIP International Federation for Information Processing, Vol. 312, pp. 370-381, 2007.

\section{Authors}

Dr. Hefnawi is currently a professor and the Chair of Graduate Studies Committee in the Department of Electrical and Computer Engineering at the Royal Military College of Canada. Dr. Hefnawi is a licensed professional engineer in the province of Ontario. He is a contributing author of several refereed journal, book chapters, and proceeding papers in the areas of wireless communications. His research interest includes cognitive radio, wireless sensor network, massive MIMO, cooperative MIMO, multiuser MIMO, and smart grid

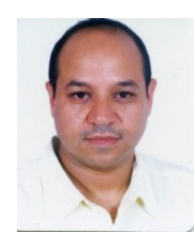
communications. 